\title{
ADAPTAÇÕES NA MASTIGAÇÃO, DEGLUTIÇÃO E FONOARTICULAÇÃO EM IDOSOS DE INSTITUIÇÃO DE LONGA PERMANÊNCIA
}

\section{Chew, deglutition and speech adaptations in aged people at a long permanence institution}

\author{
Renata Milena Freire Lima ${ }^{(1)}$, Ana Karênina de Freitas Jordão do Amaral (2), \\ Edylla Barbosa Lins Aroucha ${ }^{(3)}$, Tirza Malta Jordão de Vasconcelos ${ }^{(4)}$, \\ Hilton Justino da Silva ${ }^{(5)}$, Daniele Andrade da Cunha ${ }^{(6)}$
}

\begin{abstract}
RESUMO
Objetivo: identificar adaptações existentes nas funções estomatognáticas de mastigação, deglutição e fonoarticulação em idosos de Instituição de longa permanência. Métodos: participaram da pesquisa 34 idosos, sendo 15 do sexo feminino e 19 do sexo masculino na faixa etária de 65 a 88 anos. Foram realizadas entrevistas contendo perguntas sobre a saúde geral e alimentação do idoso e avaliações fonoaudiológicas a fim de caracterizar as funções de mastigação, deglutição e fonoarticulação. Resultados: em relação à mastigação, foi observado que a maioria dos idosos apresentou mastigação adaptada, com participação exagerada da musculatura perioral, movimentos mandibulares verticais e o padrão mastigatório predominante foi bilateral simultâneo. Em relação à deglutição, observou-se que assim como a mastigação, esta função se apresentou adaptada na maioria dos voluntários, com a participação exagerada da musculatura perioral e ruído durante a deglutição. $\mathrm{Na}$ avaliação da fonoarticulação, foi visto que mais de $50 \%$ dos idosos apresentou escape de ar durante a fala, assim como uma elevação de laringe reduzida e presença de substituição, omissão, distorção e imprecisão. Conclusão: observou-se que os idosos se adaptam durante o processo de mastigação a fim de manter sua dieta o mais próximo possível da realizada quando jovem. Também foi visto que os pesquisados realizam compensações durante a deglutição. As características fonoarticulatórias encontradas não limitam severamente a comunicação dos idosos atualmente. Foi possível ainda observar que o atendimento ao idoso necessita ser interdisciplinar e que o fonoaudiólogo pode e deve fazer parte dessa equipe auxiliando na prevenção e/ou reabilitação de problemas relacionados às funções estomatognáticas.
\end{abstract}

DESCRITORES: Mastigação; Deglutição; Fala; Idoso

(1) Fonoaudióloga Diretora da Clínica Renata Milena Freire Lima, PE; Especializanda em Motricidade Orofacial pela Faculdade Integrada do Recife.

(2) Fonoaudióloga do Centro de Reabilitação Francisco Loreiro, Jaboatão dos Guararapes, PE; Especialista em Motricidade Oral; Especialista em Gerontologia pela Universidade Federal da Paraíba; Mestranda em Patologia/Morfologia Aplicada pela Universidade Federal de Pernambuco.

(3) Aluna do curso de Graduação em Fonoaudiologia da Faculdade Integrada do Recife, FIR, Recife, PE.

(4) Aluna do curso de Graduação em Fonoaudiologia da Faculdade Integrada do Recife, FIR, Recife, PE.

(5) Fonoaudiólogo; Docente da Faculdade Integrada do Recife, FIR, Recife, PE; Mestre em Morfologia; Doutor em Nutrição pela Universidade Federal de Pernambuco.

\section{INTRODUÇÃO}

O envelhecimento, atualmente, apresenta-se como um fenômeno universal. No Brasil, de acordo com a Organização Mundial de Saúde, estima-se que até o ano de 2025, a população idosa crescerá 16 vezes, contra cinco da população total. Esses

(6) Fonoaudióloga; Coordenadora do curso de Fonoaudiologia da Faculdade Integrada do Recife, FIR, Recife, PE; Mestre e Doutoranda em Nutrição pela Universidade Federal de Pernambuco.

Projeto financiado pelo programa PIBIC/FACEPE/FIR Governo do Estado de Pernambuco.

Conflito de interesses: inexistente 
dados classificam o Brasil como o país que possui a sexta população do mundo em idosos, correspondendo a mais de 32 milhões de pessoas com idade igual ou superior a 60 anos, o que corresponde a $15,1 \%$ da população ${ }^{1}$. Junto às modificações observadas na pirâmide populacional, as doenças próprias do envelhecimento ganham maior expressão dentro da sociedade. Em geral, essas doenças nos idosos são crônicas e múltiplas, e exigem um acompanhamento constante por parte de vários profissionais da área de saúde ${ }^{2}$.

O processo de envelhecimento da população possui como determinantes, as taxas de fecundidade, natalidade e mortalidade. O declínio dessas desempenha papel (éis) muito importante(s) na determinação do tempo e da velocidade em que o envelhecimento ocorre ${ }^{3,4}$.

Observa-se também mudança no modelo familiar, surgindo, dessa forma, uma limitação no tamanho da família, que antes tinha média de seis a sete filhos, reduzindo para dois ou três no máximo. Nesse sentido, as famílias se deparam com diversas dificuldades, muitas vezes não compreendidas. Embora algumas famílias se mantenham como as mais tradicionais, cuidando e acolhendo seus parentes mais velhos, outras se modificam e surge a questão de quem é responsável por cuidar do idoso ${ }^{5}$

Nesse momento, a família começa a questionar a necessidade de uma instituição que possa cuidar desse(a) idoso(a). Afinal, alguns idosos requerem acompanhamento durante um tempo bastante significativo e cuidados contínuos. Dúvidas como: "com quem esse idoso irá ficar dentro de casa? Como fazer se filhos e/ou netos precisam trabalhar, estudar? Que riscos poderia está correndo se ficar sozinho?" 4,5, surgem nesse momento.

As atuais instituições asilares têm como proposta acolher e, cuidar de idosos e prestam serviços diversos, dentre os quais: o Serviço de Residência, onde o idoso pode ficar permanentemente na instituição dispondo de local para dormir, refeições, medicamentos, acompanhado por uma equipe especializada no atendimento de pessoas idosas, podendo participar das atividades promovidas pela instituição, recebendo visitas de familiares. Já no serviço Centro Dia o idoso pode dispor das atividades realizadas pela instituição como artesanato, pintura, música, entre outras durante o dia, tendo o direito também de realizar todas as refeições e no final do dia um responsável leva o idoso para dormir em casa ${ }^{3}$.

Diante de tudo isso, é possível questionar: qual a relação direta com a Fonoaudiologia? Como resposta tem-se: campo de trabalho. É fundamental o trabalho fonoaudiológico que pode ser prestado ao idoso em instituição. O envelhecimento apresenta como características próprias uma série de modificações morfofisiológicas, dentre estas, modificações da motricidade orofacial, da voz, da audição, da linguagem, enfim, do campo de atuação da Fonoaudiologia, que é a comunicação humana ${ }^{6,7}$.

Em relação à Motricidade Orofacial, pode existir uma série de adaptações tanto nas estruturas, envolvendo mandíbula, área oclusal, lábios, língua, bochechas, palato, como nas funções estomatognáticas: sucção, mastigação, respiração, deglutição e fonoarticulação, podendo essas adaptações comprometer a qualidade de vida, uma vez que interferem nas atividades cotidianas de vida diária como a comunicação do idoso e até mesmo funções importantes como a respiração, mastigação e deglutição e fonoarticulação, vitais para o ser humano ${ }^{8}$.

$\mathrm{Na}$ mastigação dos idosos podem-se encontrar adaptações, por conta da diminuição do tônus e força da língua, que prejudica a coordenação motora na lateralização do bolo alimentar durante o ato mastigatório ${ }^{9,10}$.

Durante o processo de mastigação, a capacidade para controlar o bolo alimentar geralmente é afetada de acordo com o funcionamento dos músculos no que diz respeito à sua motilidade e dos proprioceptores orais que se relacionam com a sensação intra-oral. A partir desse momento, surge a preocupação em relação ao risco de um movimento de posteriorização do alimento resultando em um escape precoce do bolo, cuja principal consequência é o acometimento da integridade das vias respiratórias ${ }^{11}$.

Sabe-se que com o avançar da idade, ocorre a perda de papilas sobre a língua, tornando o epitélio mais delgado e consequentemente ocorre o declínio na sensação gustativa. Dessa forma, o idoso não consegue sentir como antes, o sabor dos alimentos ${ }^{12}$.

Em consequência dessas características da mastigação, têm-se uma deglutição ocorrendo também com adaptações. É necessário o esclarecimento de que uma deglutição adaptada difere de uma deglutição alterada. A primeira está relacionada à Presbifagia, que corresponde ao envelhecimento natural, fisiológico, do mecanismo de deglutição, já a segunda, relaciona-se com a Disfagia que se refere à dificuldade de coordenar os movimentos da mastigação, deglutição e respiração, tendo como principais causas: acidente vascular cerebral; trauma crânio-encefálico; câncer de cabeça e pescoço; tumores cerebrais; demências ${ }^{13}$.

Durante o processo de deglutição do idoso, sem comprometimento neurológico, é possível observar uma elevação laríngea reduzida e a presença de resíduos de alimentos após a deglutição. Também 
é comum a diminuição da motilidade dos músculos faríngeos, disfunção da ação da cartilagem epiglótica, que diminuem a eficácia dos mecanismos de proteção da via aérea aumentando a possibilidade de penetração laríngea ${ }^{10}$.

No idoso, é possível observar alterações em sua comunicação em consequência da redução dos níveis de consciência, atenção, memória, raciocínio e fala. Também podem ser encontradas alterações musculares que comprometem os órgãos fonoarticulatórios e consequentemente alteram a inteligibilidade de fala ${ }^{14}$.

Além das modificações anatômicas e/ou funcionais já supracitadas, a fonoarticulação pode também ser afetada pela ausência de dentes, problemas periodontais, atrofia dos músculos mastigatórios, uso de prótese dentária e diminuição da produção de saliva ${ }^{15}$.

Dessa forma, o objetivo desse estudo é identificar quais as adaptações presentes nas funções estomatognáticas de mastigação, deglutição e fonoarticulação em idosos de Instituição de longa permanência.

\section{MÉTODOS}

A pesquisa ocorreu em uma instituição de longa permanência, situada no bairro de Cavaleiro na cidade de Jaboatão dos Guararapes, estado de Pernambuco. A instituição abriga 129 idosos, com idade igual ou superior a 65 anos. Possui dois paviIhões, um feminino e outro masculino. A instituição possui 64 anos de funcionamento e dispõe de uma equipe formada por fisioterapeutas, psicólogos, médico e assistente social.

Foram incluídos como voluntários 34 idosos com idade entre 65 e 88 anos, todos residentes da Instituição onde a pesquisa foi desenvolvida. Foram excluídos da pesquisa:

- os internos com histórico de alterações neurológicas, tais como acidente vascular cerebral isquêmico ou hemorrágico; trauma cranioencefálico; Doença de Parkinson; Doença de Alzheimer;

- aqueles que não se alimentavam por via oral, pois para a avaliação da mastigação e deglutição foram utilizados alimentos sólidos, pastosos e líquidos.
- indivíduos com sinais e/ou sintomas de perda auditiva que comprometesse a comunicação, visto que na avaliação da fonoarticulação, era necessária a escuta e compreensão do comando solicitado.

A coleta de dados ocorreu no período de Agosto a Novembro de 2006. Todos os procedimentos foram realizados na própria instituição. Esta pesquisa caracteriza-se por ser um estudo do tipo descritivo, observacional e transversal.

Como método de coleta realizou-se primeiramente uma entrevista elaborada pelas pesquisadoras composta de perguntas relacionadas à saúde geral, alimentação atual e aspectos da mastigação, deglutição e fonoarticulação do idoso com base em Bilton, Tega, Soares, 1997 e Junqueira, 1998 (Figura 1).

Em seguida, as pesquisadoras realizaram uma avaliação, que contemplou os seguintes aspectos: postura, morfologia, tonicidade e mobilidade das estruturas estomatognáticas e o desempenho das funções de mastigação, deglutição e fonoarticulação (Figura 2).

Durante a avaliação das estruturas orais, foram utilizadas luvas e espátulas. Objetivando avaliar as funções de mastigação e deglutição, foram utilizados: pão francês de 50 gramas, iogurte de consistência pastosa e água mineral em temperatura ambiente.

Para a realização da avaliação da função de fonoarticulação foi utilizado um álbum articulatório confeccionado para a pesquisa com várias imagens contemplando todos os fonemas nas três posições distintas, (início, meio e final da palavra). Nessa etapa houve a necessidade de gravação para posterior análise detalhada.

O projeto foi aprovado pelo Comitê de Ética em Pesquisa da FIR - Faculdade Integrada do Recife, sob CAAE no 0935.0.000.100-06.

Utilizou-se a análise descritiva para expor os resultados obtidos através do instrumento de coleta. A apresentação das variáveis mensuradas foi feita por meio de tabelas, incluindo também o uso de algumas medidas descritivas (mínimo, máximo, média e desvio padrão). O software utilizado foi o Excel 2000. 
Nome:

Sexo:
PROTOCOLO DE ENTREVISTA

Adaptado de SUZUKI (1997) e JUNQUEIRA (1998).

dade:

2. Dados sobre saúde Geral atual:

\begin{tabular}{|c|c|c|}
\hline 2.1 Hipertensão & $1($ ) Sim & 2 ( ) Não \\
\hline 2.2 Diabetes & 1() $\mathrm{Sim}$ & 2 ( ) Não \\
\hline 2.3 Cardiopatia & 1( ) Sim & 2 ( ) Não \\
\hline 4 Doenças pulmonares & 1( ) Sim & ) Não \\
\hline 2.5 Refluxo gastroesofágico & 1 ( ) Sim & Não \\
\hline 2.6 Outros & $1($ ) Sim & 2 ( ) Não \\
\hline
\end{tabular}

3. Fatores associados:

3.1 Fez ou faz uso de cigarro 1( ) Sim 2( ) Não

3.2 Fez ou faz uso de álcool 1( ) Sim 2 ( ) Não

$\begin{array}{lll}3.3 \text { Outros } & 1(\text { ) Sim } & 2(\text { ) Não }\end{array}$

4. Dados sobre alimentação atual

4.1 Tipo de alimento ingerido:

1( ) Sólido 2 ( ) Pastoso 3 ( ) Líquido

5. Queixas sobre o processo de alimentação - Mastigação

5.1 Quantidade de refeições durante o dia:

1( ) uma 2( ) duas 3( ) três

5.2 Necessita de temperar mais a comida para perceber o sabor:

1 ( ) Sim 2 2( ) Não $\quad 3($ ) Às vezes

5.3 Presença de dificuldade em mastigar algum alimento:

1 ( ) Sim 2( ) Não

5.4 Presença de dor durante a mastigação:

1 ( ) Sim 2( ) Não $3($ ) Às vezes

5.5 Presença de cansaço para mastigar algum alimento:
1( ) Sim
2 ( ) Não
3 ( ) Às vezes

6. Queixas sobre o processo de alimentação - Deglutição

6.1 Presença de engasgo durante as refeições:

1 ( ) Sim 2 2( ) Não 3 3( ) Às vezes

6.2 Presença de engasgo após as refeições:

1 ( ) Sim 2( ) Não $3\left(\begin{array}{ll}\text { ) Às vezes } \\ \text { 2 }\end{array}\right.$

6.3 Presença de tosse durante as refeições:

1 ( ) Sim 2( ) Não $3($ ) Às vezes

6.4 Presença de tosse após as refeições:

1 ( ) Sim 2( ) Não $3($ ) Às vezes

6.5 Presença de cansaço durante as refeições:

1 ( ) Sim 2( ) Não 3 3( ) Às vezes

6.6 Presença de cansaço após as refeições:

1( ) Sim 2 ( ) Não 3 ( ) Às vezes

6.7 Sensação de comida "parada" na garganta:

1 ( ) Sim 2( ) Não $\quad 3($ ) Às vezes

6.8 Ardor e/ou dor na garganta durante ou após a refeição:

1( ) Sim 2 ( ) Não 3 ( ) Às vezes

6.9 Necessidade de auxiliar a descida do alimento com líquido:

1( ) Sim 2 ( ) Não $\quad 3($ ) Às vezes

6.10 Presença de empanzinamento durante e/ou após a refeição:

1( ) Sim 2( ) Não $\quad 3($ ) Às vezes

7. Queixas sobre a fonoarticulação

7.1 Sente alguma dificuldade em falar alguma palavra:

1( ) Sim 2( ) Não $\quad 3$ ( ) Às vezes

7.2 Sente a "boca travada" em algum momento durante a fala:

1( ) Sim 2 ( ) Não $\quad 3($ ) Às vezes

7.3 Percebe sua fala mais lenta:

1( ) Sim 2 ( ) Não $\quad 3($ ) Às vezes

7.4 Percebe sua fala mais rápida:

1 ( ) Sim 2( ) Não $\quad 3($ ) Às vezes

7.5 Percebe maior escape de ar durante a fala:
1 ( ) Sim
2 ( ) Não
7.6 Outros
1( ) Sim
3 ( ) Às vezes
2 ( ) Não

5 ( ) Todas as consistências

4 ( ) quatro

5 ( ) cinco

Figura 1 - Protocolo de anamnese Adaptado de SUZUKI (1997) e JUNQUEIRA (1998) 


\section{PROTOCOLO DE AVALIAÇÃO \\ Adaptado de: SUZUKI (1997) e JUNQUEIRA (1998).}

Dados Pessoais:

Nome:

Sexo:

Idade:

Avaliação das estruturas estomatognáticas no que diz respeito à morfologia e postura

\section{1- LÁBIOS}

1.1 ( ) Fechados

1.2( ) Entreabertos

1.3 ( ) Possibilidade de vedamento

1.4 ( ) Dificuldade de vedamento

1.5 ( ) Superior encurtado

1.6 ( ) Inferior evertido

1.7 ( ) Superior fino

1.8 ( ) Inferior fino

1.9 ( ) Superior e inferior finos

\section{2 - LINGUA}

2.1 ( ) Normal

2.2( ) Alargada

2.3 ( ) Sem ponta

2.4 ( ) Marcas nas laterais

2.5 ( ) Posicionada na região da papila palatina

2.6 ( ) Posicionada na região do assoalho bucal

2.7 ( ) Posicionada entre os dentes

2.8 ( ) Frênulo normal

2.9 ( ) Frênulo curto

\section{3-BOCHECHAS}

3.1 ( ) Simétricas

3.2( ) Assimétricas

3.3 ( ) Marcas ou ferimentos internamente

3.4 ( ) Direita mais avolumada

3.5 ( ) Esquerda mais avolumada

3.6 ( ) Ambas avolumadas

3.7 ( ) Direita caída

3.8 ( Esquerda caída

3.9 ( ) Ambas caídas

4-DENTIÇÃO

4.1 Número de dentes superiores:

4.2 Número de dentes inferiores:

4.3 ( ) Bom estado de conservação

4.4 ( ) Regular estado de conservação

4.5 ( ) Mau estado de conservação

\section{5 - PRÓTESE}

5.1 ( ) Não faz uso de prótese

5.2 ( ) Faz uso de prótese parcial superior

5.3 ( ) Faz uso de prótese parcial inferior

5.4 ( ) Faz uso de prótese total inferior

5.5 ( ) Faz uso de prótese total superior

\section{6 - PALATO DURO}

6.1 ( ) Normal
6.2 ( ) Ogival
6.3 ( ) Atrésico

7 - PALATO MOLE

7.1 ( ) Normal

7.2 ( ) Úvula bífida 


\section{Avaliação das estruturas estomatognáticas no que diz respeito à tonicidade}

\section{1 - LÁBIOS}

1.1 ( ) Tônus normal

1.2 ( ) Hipofuncionantes

1.3 ( ) Hiperfuncionantes

1.4 ( ) Superior hipofuncionante

1.5 ( ) Superior hiperfuncionante

1.6 ( ) Inferior hipofuncionante

1.7 ( ) Inferior hiprfuncinante

\section{2 - LÍNGUA}

2.1 ( ) Tônus normal

2.2( ) Hipotensa

2.3 ( ) Hipertensa

\section{3-BOCHECHAS}

3.1 ( ) Tônus normal

3.2 ( ) Direita hipofuncionante

3.3 ( ) Direita hiperfuncionante

3.4 ( ) Esquerda hipofuncionante

3.5 ( ) Esquerda hiperfuncionante

\section{4 - MENTUAL}

4.1 ( ) Normal

4.2 ( ) Hipofuncionante

4.3( )Hiperfuncionante

\section{Avaliação das estruturas estomatognáticas no que diz respeito à mobilidade}

\section{1- LÁBIOS}
1.1 ( ) Normal
1.2 ( ) Adaptado
1.3 ( ) Alterado
1.4 ( ) Tremor

\section{2 - LÍNGUA}

2.1 ( ) Normal

2.2 ( ) Adaptada

2.3 ( ) Alterada

2.4 ( ) Tremor

\section{3-MANDÍBULA}

\section{1 ( ) Normal}

3.2 ( ) Desvio na abertura para direita

3.3 ( ) Desvio na abertura para esquerda

3.4 ( ) Desvio no fechamento para direita

3.5 ( ) Desvio no fechamento para esquerda

3.6 ( ) Creptações

3.7 ( ) Estalidos

\section{4 - PALATO MOLE}

4.1 ( ) Bom funcionamento velo faríngeo

4.2 ( ) Déficit de funcionamento velo faríngeo 


\section{Avaliação das funções estomatognáticas: mastigação; deglutição e fonoarticulação}

\section{1- MASTIGAÇÃO}

1.1 ( ) Bilateral alternada

1.2 ( ) Bilateral alternada com predomínio do lado direito

1.3 ( ) Bilateral alternada com predomínio do lado esquerdo

1.4 ( ) Bilateral simultânea

1.5 ( ) Bilateral simultânea com predomínio do lado direito

1.6 ( ) Bilateral simultânea com predomínio do lado esquerdo

1.7 ( ) Unilateral direita

1.8 ( ) Unilateral esquerda

1.9 ( ) Adaptada

1.10 ( ) Alterada

1.11 ( ) Lenta

1.12 ( ) Rápida

1.13 ( ) Ruidosa

1.14 ( ) Lábios fechados

1.15 ( ) Lábios abertos

1.16 ( ) Interposição de lábio inferior

1.17 ( ) Participação exagerada da musculatura perioral

1.18 ( ) Movimentos rotatórios de mandíbula

1.19 ( ) Movimentos verticais de mandíbula

\section{2 - DEGLUTIÇÃO}

2.1 ( ) Normal

2.2( ) Alterada

2.3 ( ) Projeção anterior de língua

2.4 ( ) Projeção lateral de língua

2.5 ( ) Interposição de lábio inferior

2.6 ( ) Participação exagerada da musculatura perioral

2.7 ( ) Projeção de cabeça

2.8 ( ) Engasgo

2.9 ( ) Ruidosa

\section{3 - FONOARTICULAÇÃO}

3.1 ( ) Normal

3.2 ( ) Lenta

3.3 ( ) Rápida

3.4 ( ) Escape de ar (sigmatismo)

3.5 ( ) Interposição de língua anterior (ceceio)

3.6 ( ) Acúmulo de saliva nas comissuras

3.7 ( ) Elevação de laringe normal

3.8 ( )Elevação de laringe reduzida

3.9 ( ) Presença de substituição

3.10 ( ) Presença de omissão

3.11 ( ) Presença de distorção

3.12 ( ) Presença de imprecisão

Figura 2 - Protocolo de avaliação Adaptado de: SUZUKI (1997) e JUNQUEIRA (1998)

\section{RESULTADOS}

A Tabela 1 apresenta a distribuição dos idosos quanto às características gerais. A média de idade foi de 74 anos. Observou-se ainda que a maioria é do sexo masculino $(55,9 \%, n=19)$. Observou-se que $50,0 \%(n=17)$ dos idosos pesquisados referiram ingerir alimentos de todas as consistências. Em relação à quantidade de refeições realizadas durante o dia, metade dos voluntários $(50,0 \%, \mathrm{n}=17)$ afirmaram realizar quatro refeições ao dia.

A Tabela 2 apresenta dados referentes à saúde geral dos sujeitos. Foi visto que $41,2 \%(n=14)$ referiram ter hipertensão; $29,4 \%(n=10)$ afirmaram ter refluxo gastroesofágico; $11,8 \%(n=4)$ se queixaram de diabetes; $8,8 \%(n=3)$ citaram cardiopatia e 8,8\% $(n=3)$ referiram doenças pulmonares.

A Tabela 3 relaciona as queixas no processo de alimentação. $\mathrm{Na}$ amostra estudada 29,4\% ( $n=10)$ 
dos idosos referem a necessidade de tempero para poder sentir o sabor da comida; 35,3\% ( $n=12)$ sentem dificuldade em mastigar, $11,8 \%(n=4)$ têm a sensação de comida "parada" na garganta e mais da metade dos voluntários $(52,9 \% n=18)$ afirmou que necessitam do auxílio de líquido para deglutir o alimento.

A Tabela 4 refere-se às queixas sobre a fonoarticulação e observou-se que apenas 17,6\% (n=6) referiram ter dificuldade em falar alguma palavra, porém não especificaram qual(is) seriam e $29,4 \%$ $(n=10)$ percebem maior escape de ar durante a fala.

As Tabelas 5 e 6 distribuem dados quanto à avaliação da morfologia e foi observado que em relação aos lábios, 58,8\% $(n=20)$ conseguem manter vedamento labial. Foi ainda observado que $8,8 \%$ $(n=3)$ possuem lábio inferior evertido, 8,8\% $(n=3)$ apresentam lábio superior fino e 2,9\% $(n=1)$ possui lábios superior e inferior finos. Quanto à língua, observou-se que $44,1 \%(n=15)$ apresentou alargamento dessa estrutura é que $76,5 \%(n=26)$ apresentaram frênulo lingual normal, fator importante para o desempenho das funções avaliadas nesta pesquisa. Avaliando-se as bochechas, percebe-se que uma quantidade significativa dos idosos apresentou simetria dessas estruturas $61,8 \% \quad(n=21)$, porém, desses, $55,9 \%(n=19)$ possuem ambas caídas, revelando hipofunção. E em relação ao palato duro foi observado que em $85,3 \%(n=29)$ são normais e em $14,7 \%$ ( $n=5)$ são ogivais.

No que se refere à dentição, foi observado que, o número médio de dentes na arcada superior é de 2,3 $\pm 3,9$ e na arcada inferior é de $2,6 \pm 4,2$. O dado torna-se mais negativo quando associado ao estado de conservação dentária, pois nenhum dos voluntários apresentou bom estado de conservação dentária, sendo este em 14,7\% (n=5) regular e $29,4 \%(n=10)$ mau de acordo com a avaliação perceptual realizada pela pesquisadora. Em relação às próteses, temos que a grande maioria dos idosos $70,6 \%$ ( $n=24$ ) não usam próteses e que $26,5 \%$ $(n=9)$ utilizam apenas na arcada inferior e $29,4 \%$ $(n=10)$ na superior.

Quanto ao palato duro, independente do uso ou não de prótese, foi observado que a grande maioria apresentou-se normais, o que corresponde a $85,3 \%$ $(n=29)$ e em relação à morfologia e a funcionalidade do palato mole, $100 \%(n=34)$ estas também apresentaram-se normais.

A Tabela 7 descreve dados que dizem respeito à avaliação da tonicidade. Os lábios de 70,6\% (n=24) apresentaram-se hipofuncionantes e apenas 2,9\% $(\mathrm{n}=1)$ apresentou-se hiperfuncionante. A língua de $79,4 \%(n=27)$ encontra-se hipotensa.

Em relação às bochechas, também se percebe o predomínio da hipofunção, $73,5 \%(n=25)$ apresentaram a direita e $76,5 \%(n=26)$ têm a esquerda hipofuncionante. E em relação ao músculo mentual, $76,5 \%(n=26)$ são hiperfuncionantes, o que provavelmente se relaciona com a hipofunção das outras estruturas já citadas e com compensações realizadas no repouso e/ou função.

A Tabela 8 relaciona os achados sobre a avaliação da mobilidade. Verifica-se que em relação aos lábios, 44,1\% ( $n=15)$ apresentam tremor, e em relação à língua, também foi possível perceber que $52,9 \%(n=18)$ apresentaram tremor durante a execução dos movimentos solicitados. No que diz respeito à mandíbula, $47,1 \%(n=16)$ são normais, ou seja, sem sinais de alterações, $17,6 \% \quad(n=6)$ apresentaram desvio na abertura para direita e $8,8 \%(n=3)$ apresentaram desvio na abertura para esquerda, $11,8 \% \quad(n=4)$ possuem crepitações e $20,6 \%(n=7)$ apresentaram estalidos durante os movimentos mandibulares realizados. E em relação ao palato mole, $64,7 \%(n=22)$ têm bom funcionamento velo faríngeo.

A Tabela 9 apresenta a distribuição dos idosos de acordo com a avaliação da função de mastigação, dessa forma é possível observar que o padrão predominante foi o bilateral simultâneo em 26,5\% $(n=9) ; 70,6 \%(n=24)$ dos idosos apresentaram uma mastigação adaptada provavelmente por conta da hipotonia da musculatura mastigatória observada nos mesmos, sendo mais frequentes adaptações como: movimentos verticais durante o processo mastigatório e participação exagerada da musculatura perioral, esta última foi observada em $82,4 \%$ $(n=28)$ da população estudada.

A Tabela 10 refere-se à avaliação das funções estomatognáticas de deglutição e fonoarticulação percebeu-se que em relação à deglutição em $88,2 \%(n=30)$ apresentou de forma adaptada, nesses idosos observou-se que houve um esforço maior para deglutir e compensações como: projeção de cabeça e uso excessivo da musculatura perioral, fatores que se relacionam com a própria mastigação adaptada e/ou à hipofunção da região orofaríngea. E por fim quanto à fonoarticulação observou-se escape de ar em 52,9\% ( $n=18), 58,8 \%$ $(n=20)$ apresentam elevação de laringe reduzida, $58,8 \%(n=20)$ apresentaram substituição, 88,2\% $(n=30)$ omissão, $55,9 \%(n=19)$ distorção e $88,2 \%$ $(n=30)$ apresentaram imprecisão. 
Tabela 1 - Distribuição de idosos de instituição de longa permanência quanto às características gerais

\begin{tabular}{lcc}
\hline Características Gerais & N & $\%$ \\
\hline Faixa Etária & 19 & 55,9 \\
65 a 74 & 15 & 44,1 \\
acima de 75 & & \\
& & 55,9 \\
Sexo & 19 & 44,1 \\
Masculino & 15 & \\
Feminino & & 14,7 \\
& & 32,4 \\
Tipo de Alimento Ingerido & 5 & 2,9 \\
Sólido & 11 & 50,0 \\
Pastoso & 1 & \\
Líquido engrossado & 17 & 20,6 \\
Todas as consistências & & 50,0 \\
& & 29,4 \\
Quantidade de refeições durante o dia & 7 & \\
Três & 17 & \\
Quatro & 10 & \\
Cinco & & \\
\hline
\end{tabular}

Tabela 2 - Distribuição de idosos de Instituição de longa permanência quanto à saúde geral

\begin{tabular}{lcccc}
\hline \multirow{2}{*}{ Dados Sobre Saúde Geral Atual } & \multicolumn{2}{c}{ Sim } & \multicolumn{2}{c}{ Não } \\
\cline { 2 - 5 } & $\mathbf{N}$ & $\%$ & $\mathbf{N}$ & $\%$ \\
\hline Hipertensão & 14 & 41,2 & 20 & 58,8 \\
Refluxo gastroesofágico & 10 & 29,4 & 24 & 70,6 \\
Diabetes & 4 & 11,8 & 30 & 88,2 \\
Cardiopatia & 3 & 8,8 & 31 & 91,2 \\
Doenças pulmonares & 3 & 8,8 & 31 & 91,2 \\
Outros & 8 & 23,5 & 26 & 73,5 \\
& & & & \\
Fatores Associados & & & & \\
Cigarro & 13 & 38,2 & 21 & 61,8 \\
Álcool & 5 & 14,7 & 29 & 85,3 \\
Outros & 0 & - & 34 & 100,0 \\
\hline
\end{tabular}

\section{DISCUSSÃO}

Durante a entrevista realizada verificou-se que $50 \%(n=17)$ dos idosos ingerem alimentos de todas as consistências, porém dessa porcentagem, $41,2 \%(n=14)$ referem ter maior controle com alimentos pastosos. Esse achado condiz com dados da literatura quando se afirma que as modificações nos hábitos alimentares surgem de forma gradativa, como forma de adaptação às situações de perdas com que os idosos se deparam, tais como a diminuição da saúde orgânica e a integridade do sistema estomatognático, no que se refere a sua morfologia e integridade ${ }^{10}$.

Em relação ao número de refeições realizadas por dia foi visto que $50 \%(n=17)$ realizam quatro refeições ao dia. Em pesquisa realizada em 2004 foi observado que $50 \%(n=5)$ realizavam cinco 
Tabela 3 - Distribuição de idosos de Instituição de longa permanência quanto às queixas sobre o processo de alimentação

\begin{tabular}{lcccccc}
\hline \multirow{2}{*}{ Queixas sobre o processo de alimentação } & \multicolumn{2}{c}{ Sim } & \multicolumn{2}{c}{ Não } & \multicolumn{2}{c}{ Às vezes } \\
\cline { 2 - 7 } & $\mathbf{N}$ & $\%$ & $\mathbf{N}$ & $\%$ & $\mathbf{N}$ & $\%$ \\
\hline Mastigação & & & & & & \\
Necessita de tempero para sentir sabor & 10 & 29,4 & 21 & 61,8 & 3 & 8,8 \\
Dificuldade em mastigar & 12 & 35,3 & 21 & 61,8 & 1 & 2,9 \\
Dor durante a mastigação & 2 & 5,9 & 31 & 91,2 & 1 & 2,9 \\
Cansaço para mastigar & 0 & - & 31 & 91,2 & 3 & 8,8 \\
& & & & & & \\
Deglutição & & & & & & \\
Engasgo durante as refeições & 3 & 8,8 & 22 & 64,7 & 9 & 26,5 \\
Engasgo após as refeições & 1 & 2,9 & 32 & 94,1 & 1 & 2,9 \\
Tosse durante as refeições & 2 & 5,9 & 25 & 73,5 & 7 & 20,6 \\
Tosse após as refeições & 0 & - & 32 & 94,1 & 2 & 5,9 \\
Cansaço durante as refeições & 0 & - & 32 & 94,1 & 2 & 5,9 \\
Cansaço após as refeições & 0 & - & 33 & 97,1 & 1 & 2,9 \\
Sensação de comida "parada" na garganta & 4 & 11,8 & 23 & 67,6 & 7 & 20,6 \\
Ardor ou dor na garganta durante ou após as refeições & 1 & 2,9 & 29 & 85,3 & 4 & 11,8 \\
Necessidade de líquido para deglutir & 18 & 52,9 & 11 & 32,4 & 5 & 14,7 \\
Empanzinamento durante ou após a refeições & 3 & 8,8 & 25 & 73,5 & 6 & 17,6 \\
\hline
\end{tabular}

Tabela 4 - Distribuição de idosos de Instituição de longa permanência quanto às queixas sobre a fonoarticulação

\begin{tabular}{lcccccc}
\hline \multirow{2}{*}{ Queixas sobre a fonoarticulação } & \multicolumn{2}{c}{ Sim } & \multicolumn{2}{c}{ Não } & \multicolumn{2}{c}{ Às vezes } \\
\cline { 2 - 7 } & $\mathbf{N}$ & $\%$ & $\mathbf{N}$ & $\%$ & $\mathbf{N}$ & $\%$ \\
\hline Dificuldade em falar alguma palavra & 6 & 17,6 & 23 & 67,6 & 5 & 14,7 \\
Sente a "boca travada" em algum momento durante a fala & 6 & 17,6 & 25 & 73,5 & 3 & 8,8 \\
Fala lentificada & 8 & 23,5 & 23 & 67,6 & 3 & 8,8 \\
Fala rápida & 6 & 17,6 & 23 & 67,6 & 5 & 14,7 \\
Maior escape de ar durante a fala & 10 & 29,4 & 23 & 67,6 & 1 & 2,9 \\
Outros & 1 & 2,9 & 33 & 97,1 & 0 & - \\
\hline
\end{tabular}

refeições ao dia. Considerando que esse estudo foi desenvolvido em uma instituição de longa permanência particular o que difere um pouco da realidade da instituição atualmente avaliada ${ }^{16}$.

Ainda durante a entrevista, no que diz respeito à saúde geral foi relatado pelos voluntários que $41,2 \%(n=14)$ possuem hipertensão, 29,4\% $(n=10)$ apresentam refluxo gastroesofágico, $11,8 \%(n=4)$ diabetes, 8,8\% $(n=3)$ cardiopatia e 8,8\% $(n=3)$ doenças pulmonares. Também foi relatada em pesquisa realizada em 2005 que a doença mais citada entre os idosos foi a hipertensão $42,1 \%(n=8){ }^{17}$.

Tem-se ainda que $38,2 \%(n=13)$ utilizam cigarros, pode-se considerar um número elevado se for levada em consideração a idade dos pesquisados. É citado em literatura que o uso prolongado de produtos provenientes do tabaco pode levar a casos de câncer de boca, de laringe, de pulmão e até mesmo de pâncreas, rins e bexiga. $E$ do ponto de vista geriátrico, fumantes de longa data mantêm uma incidência mais elevada de câncer nos 20 anos seguintes após terem cessado o uso do cigarro ${ }^{18}$.

Em relação ao consumo de álcool percebe-se um número menor de $14,7 \%(n=5)$, o que condiz com estudo que cita que a prevalência dos problemas da bebida alcoólica na população tende a declinar com o avançar da idade ${ }^{19}$. 
Tabela 5 - Distribuição de idosos de Instituição de longa permanência quanto à avaliação da morfologia: lábios, língua e bochechas

\begin{tabular}{|c|c|c|c|c|}
\hline \multirow{2}{*}{ Morfologia } & \multicolumn{2}{|c|}{ Sim } & \multicolumn{2}{|c|}{ Não } \\
\hline & $\mathbf{N}$ & $\%$ & $\mathbf{N}$ & $\%$ \\
\hline \multicolumn{5}{|l|}{ Lábios } \\
\hline Fechados & 20 & 58,8 & 14 & 41,2 \\
\hline Entreabertos & 14 & 41,2 & 20 & 58,8 \\
\hline Entreaberto com possibilidade de vedamento & 8 & 23,5 & 26 & 76,5 \\
\hline Dificuldade de vedamento & 4 & 11,8 & 30 & 88,2 \\
\hline Superior encurtado & 0 & - & 34 & 100,0 \\
\hline Inferior evertido & 3 & 8,8 & 31 & 91,2 \\
\hline Superior fino & 3 & 8,8 & 31 & 91,2 \\
\hline Inferior fino & 0 & - & 34 & 100,0 \\
\hline Superior e inferior finos & 1 & 2,9 & 33 & 97,1 \\
\hline \multicolumn{5}{|l|}{ Língua } \\
\hline Normal & 10 & 29,4 & 24 & 70,6 \\
\hline Alargada & 15 & 44,1 & 19 & 55,9 \\
\hline Marcas nas laterais & 2 & 5,9 & 32 & 94,1 \\
\hline Posicionada na região da papila palatina & 0 & - & 34 & 100,0 \\
\hline Posicionada na região do assoalho bucal & 5 & 14,7 & 29 & 85,3 \\
\hline Posicionada entre os dentes & 0 & - & 34 & 100,0 \\
\hline Freio normal & 26 & 76,5 & 8 & 23,5 \\
\hline Freio curto & 3 & 8,8 & 31 & 91,2 \\
\hline \multicolumn{5}{|l|}{ Bochechas } \\
\hline Simétricas & 21 & 61,8 & 13 & 38,2 \\
\hline Assimétricas & 13 & 38,2 & 21 & 61,8 \\
\hline Marcas ou ferimentos internamente & 2 & 5,9 & 32 & 94,1 \\
\hline Direita mais avolumada & 7 & 20,6 & 27 & 79,4 \\
\hline Esquerda mais avolumada & 6 & 17,6 & 28 & 82,4 \\
\hline Ambas avolumadas & 3 & 8,8 & 31 & 91,2 \\
\hline Direita mais caída & 3 & 8,8 & 31 & 91,2 \\
\hline Esquerda mais caída & 5 & 14,7 & 29 & 85,3 \\
\hline Ambas caídas & 19 & 55,9 & 15 & 44,1 \\
\hline
\end{tabular}

Em relação às queixas sobre o processo de alimentação percebe-se que, $29,4 \%(n=10)$ necessitam temperar mais a comida para sentir o sabor. Em estudo desenvolvido em 2004, foi citado que pelo menos $50 \%(n=5)$ dos voluntários também referiram essa necessidade ${ }^{16}$. A questão de temperar mais a alimentação pode ser justificada pela diminuição do número de botões gustativos e/ou por conta da higiene oral geralmente prejudicada, o que dificulta a estimulação dos quimiorreceptores orais ${ }^{20}$.

Também foi visto que 35,3\% ( $n=12)$ têm dificuldade em mastigar algum alimento, o que condiz com o que é afirmado por autores quando citam que de forma geral, com o avanço da idade é comum acontecer a preferência por consistências mais macias e/ou pastosas a fim de facilitar o processo de alimentação ${ }^{21}$. Apenas 5,9\% $(n=2)$ possuem dor durante a mastigação e $8,8 \%(n=3)$ sentem cansaço para mastigar algum alimento às vezes, o que pode ser justificado pela afirmação em literatura de que na medida em que envelhecemos, estamos mais propensos à fadiga muscular por conta do envelhecimento e hipotonia da fibra dos músculos da mastigação ${ }^{21}$.

Sobre a deglutição observou-se que 8,8\% $(n=3)$ referem engasgos durante as refeições, assim como 
Tabela 6 - Distribuição de idosos de Instituição de longa permanência quanto à avaliação da morfologia: dentição, prótese, palato duro, palato mole

\begin{tabular}{|c|c|c|c|c|}
\hline \multirow{2}{*}{ Morfologia } & \multicolumn{2}{|c|}{ Sim } & \multicolumn{2}{|c|}{ Não } \\
\hline & $\mathrm{N}$ & $\%$ & $\mathrm{~N}$ & $\%$ \\
\hline \multicolumn{5}{|l|}{ Dentição } \\
\hline Número de dentes superiores $(2,3 \pm 3,9)$ & - & - & - & - \\
\hline Número de dentes inferiores $(2,6 \pm 4,2)$ & - & - & - & - \\
\hline Bom estado de conservação & 0 & - & 34 & 100,0 \\
\hline Regular estado de conservação & 5 & 14,7 & 29 & 85,3 \\
\hline Mau estado de conservação & 10 & 29,4 & 24 & 70,6 \\
\hline \multicolumn{5}{|l|}{ Prótese } \\
\hline Não faz uso de prótese & 24 & 70,6 & 10 & 29,4 \\
\hline Faz uso de prótese parcial superior & 0 & - & 34 & 100,0 \\
\hline Faz uso de prótese parcial inferior & 0 & - & 34 & 100,0 \\
\hline Faz uso de prótese total inferior & 9 & 26,5 & 25 & 73,5 \\
\hline Faz uso de prótese total superior & 10 & 29,4 & 24 & 70,6 \\
\hline \multicolumn{5}{|l|}{ Palato Duro } \\
\hline Normal & 29 & 85,3 & 5 & 14,7 \\
\hline Ogival & 5 & 14,7 & 29 & 85,3 \\
\hline Atrésico & 0 & - & 34 & 100,0 \\
\hline \multicolumn{5}{|l|}{ Palato Mole } \\
\hline Normal & 0 & - & 34 & 100,0 \\
\hline Úvula bífida & 0 & - & 34 & 100,0 \\
\hline
\end{tabular}

também é descrito em outra pesquisa realizada em 2004, que refere que $30 \%(n=3)$ também apresentaram essa queixa ${ }^{16}$. Foi identificado que $11,8 \%$ $(n=4)$ referem a sensação de comida "parada" na garganta, dado encontrado em pesquisa realizada em 2005, a qual afirma que $15,8 \%(n=3)$ apresentam a sensação de estase ${ }^{17}$.

Um trabalho desenvolvido em 1997 relata que indivíduos mais jovens têm esvaziamento esofágico mais rápido que os idosos, sendo observável através da videofluoroscopia. Indivíduos com idade superior a 65 anos demonstram visíveis mudanças no trato esofágico, esvaziamento lentificado como de "conta-gotas" e presença de ondas terciárias denotando o presbiesôfago, e que estes sintomas possuem relação direta com a sensação de comida "parada" na garganta ${ }^{21}$.

Um dado significativo foi que $52,9 \%(n=18)$ dos voluntários referiram a necessidade do auxílio do líquido para deglutir o alimento, 8,8\% $(n=3)$ têm empanzinamento, que é a sensação de estômago excessivamente preenchido de alimento durante ou após refeições, dados que reforçam a afirmação encontrada em literatura de que na fase faríngea é possível perceber uma redução no grau de elevação anterior da laringe e redução do peristaltismo laríngeo ${ }^{22}$.

Quanto às queixas em relação à fonoarticulação observou-se que $17,6 \%(n=6)$ apresentam dificuldade em falar alguma palavra, $17,6 \%(n=6)$ sentem a "boca travada" em algum momento durante a fala, $23,5 \%(n=8)$ percebem a fala mais lenta, $17,6 \%(n=6)$ percebem a fala mais rápida e $29,4 \%$ $(n=10)$ acreditam que apresentam maior escape de ar durante a fala. Dessa forma, percebe-se que os idosos frequentemente comunicam-se de forma efetiva com o seu meio, pois as modificações que ocorrem em sua articulação são amenizadas pelos processos de adaptação / compensação ${ }^{23}$.

No que diz respeito à avaliação da morfologia foi observado no momento da avaliação que a maior parte dos voluntários apresentou lábios fechados, porém em $41,2 \%(n=14)$ os lábios se encontravam entreabertos. Destes, $23,5 \%(n=8)$ possuíam possibilidade de vedamento e $11,8 \%(n=4)$ realizam com dificuldade. Esses fatores se relacionam com a 
Tabela 7 - Distribuição de idosos de Instituição de longa permanência quanto à avaliação da tonicidade: lábios, língua, bochechas e mentual

\begin{tabular}{|c|c|c|c|c|}
\hline \multirow{2}{*}{ Tonicidade } & \multicolumn{2}{|c|}{ Sim } & \multicolumn{2}{|c|}{ Não } \\
\hline & $\mathbf{N}$ & $\%$ & $\mathbf{N}$ & $\%$ \\
\hline \multicolumn{5}{|l|}{ Lábios } \\
\hline Tônus normal & 9 & 26,5 & 25 & 73,5 \\
\hline Hipotônicos & 24 & 70,6 & 10 & 29,4 \\
\hline Hipertônicos & 1 & 2,9 & 33 & 97,1 \\
\hline Superior hipotônico & 0 & - & 34 & 100,0 \\
\hline Superior hipertônico & 0 & - & 34 & 100,0 \\
\hline Inferior hipotônico & 0 & - & 34 & 100,0 \\
\hline Inferior hipertônico & 0 & - & 34 & 100,0 \\
\hline \multicolumn{5}{|l|}{ Língua } \\
\hline Tônus normal & 7 & 20,6 & 27 & 79,4 \\
\hline Hipofuncionante & 27 & 79,4 & 7 & 20,6 \\
\hline Hiperfuncionante & 0 & - & 34 & 100,0 \\
\hline \multicolumn{5}{|l|}{ Bochechas } \\
\hline Tônus normal & 3 & 8,8 & 31 & 91,2 \\
\hline Direita hipotônicas & 25 & 73,5 & 9 & 26,5 \\
\hline Direita hipotônicas & 2 & 5,9 & 32 & 94,1 \\
\hline Esquerda hipotônicas & 26 & 76,5 & 8 & 23,5 \\
\hline Esquerda hipertônicas & 6 & 17,6 & 28 & 82,4 \\
\hline \multicolumn{5}{|l|}{ Mentual } \\
\hline Normal & 4 & 11,8 & 30 & 88,2 \\
\hline Hipotônico & 4 & 11,8 & 30 & 88,2 \\
\hline hipertônico & 26 & 76,5 & 8 & 23,5 \\
\hline
\end{tabular}

afirmação de que o declínio do tônus pode relacionar-se à diminuição da massa muscular e a mudança morfológica que diminui as unidades motoras funcionantes ${ }^{24}$.

Em relação à língua, percebe-se que os achados se relacionam com afirmação feita em 2003, quando é citado que assim como todo o organismo a musculatura orofacial também sofre modificações com o avançar da idade ${ }^{25}$.

Durante a avaliação das bochechas, foi percebido que a maioria dos idosos avaliados apresentou bochechas simétricas e ambas caídas. Dados que corroboram o que é dito em literatura quando se afirma que há uma mudança na composição da musculatura com decréscimo do número de unidades motoras, caracterizando-as com menos fibras de rápida contração remanescentes, fazendo com que ocorra diminuição do tônus das estruturas ${ }^{10}$.

No que se refere à dentição, foi observado que o número médio de dentes na arcada superior é de
$2,3 \pm 3,9$ e na arcada inferior é de $2,6 \pm 4,2$. Dados que se relacionam com o que é citado em literatura quando é afirmado que existe uma alta incidência de cáries, doenças periodontais, somados a modificações da produção salivar associado aos maus cuidados de higiene oral ${ }^{25}$. Afirma-se que o edentulismo configura-se como resultado de medidas de atenção à saúde oral inexistente ou fracassadas e que a perda parcial e/ou total dos dentes é aceita, pela sociedade em geral, como algo normal ou natural do avanço da idade, fator negativo, mas que se faz presente na maioria dos idosos ${ }^{23}$. Os idosos institucionalizados possuem em média menos dentes naturais do que idosos que vivem independentes ${ }^{26}$.

Nenhum dos idosos pesquisados apresentou bom estado de conservação dos dentes. Em estudo realizado foi percebido que os cuidados necessários com a cavidade oral, principalmente no que diz respeito aos dentes, muitas vezes é algo esquecido, 
Tabela 8 - Distribuição de idosos de Instituição de longa permanência quanto à avaliação da mobilidade: lábios, língua, mandíbula e palato mole

\begin{tabular}{lcccc}
\hline \multirow{2}{*}{ Mobilidade } & \multicolumn{2}{c}{ Sim } & \multicolumn{2}{c}{ Não } \\
\cline { 2 - 5 } & $\mathbf{N}$ & $\%$ & $\mathbf{N}$ & $\%$ \\
\hline Lábios & & & & \\
Normal & 11 & 32,4 & 23 & 67,6 \\
Adaptada & 7 & 20,6 & 27 & 79,4 \\
Alterada & 1 & 2,9 & 33 & 97,1 \\
Tremor & 15 & 44,1 & 19 & 55,9 \\
& & & & \\
Língua & & & & \\
Normal & 7 & 20,6 & 27 & 79,4 \\
Adaptada & 8 & 23,5 & 26 & 76,5 \\
Alterada & 1 & 2,9 & 33 & 97,1 \\
Tremor & 18 & 52,9 & 16 & 47,1 \\
Mandíbula & & & & \\
Normal & & & & \\
Desvio na abertura para direita & 16 & 47,1 & 18 & 52,9 \\
Desvio na abertura para esquerda & 6 & 17,6 & 28 & 82,4 \\
Desvio no fechamento para direita & 3 & 8,8 & 31 & 91,2 \\
Desvio no fechamento para esquerda & 0 & - & 34 & 100 \\
Presença de crepitações & 0 & - & 34 & 100 \\
Presença de estalido & 4 & 11,8 & 30 & 88,2 \\
Palato Mole & 7 & 20,6 & 27 & 79,4 \\
Bom funcionamento velo- faríngeo & & & & \\
Déficit de funcionamento velo-faríngeo & & & & 35,3 \\
\hline
\end{tabular}

fator que prejudica consequentemente as funções estomatognáticas ${ }^{20}$. Em relação às próteses, grande parte dos idosos não as utiliza, salientando que no presente estudo não foi avaliada a adaptação dessas próteses. Afirma-se que é possível o surgimento de zonas de hiperqueratose nas áreas de adaptação de prótese e ainda que a mucosa oral torna-se mais propensa à ulcerações quando essas não se encontram bem adaptadas ${ }^{8,25}$.

$\mathrm{Em}$ relação à morfologia do palato duro, não foram encontradas referências em literatura sobre esta estrutura no idoso. Quanto à tonicidade observou-se que em relação aos lábios, o número de idosos que apresenta hipofunção dessa estrutura é prevalente. No que diz respeito à língua, também foi observada hipotensão na maioria dos voluntários. Avaliando-se as bochechas, mais uma vez observa-se um número significativo de bochechas hipofuncionantes.

Quanto à mobilidade, foi possível verificar que em relação aos lábios, vários idosos apresentaram déficit na realização dos movimentos solicitados. Dados que corroboram a afirmação em pesquisa realizada em 2004, que, de maneira geral, foi observada uma tendência à hipofunção da musculatura e certa dificuldade em realizar alguns movimentos solicitados de lábios, língua e bochechas ${ }^{16}$. Dados que irão relacionar-se diretamente com o desempenho das funções estomatognáticas de mastigação, deglutição e fonoarticulação, pois essas irão depender do funcionamento dos músculos tanto no que diz respeito à sua motilidade, como sua sensação ${ }^{11}$.

Em relação à mandíbula, foi visto que $20,6 \%$ dos pesquisados possuem estalidos, sendo este item avaliado está próximo ao coletado em pesquisa de 2004 , em que foi descrito que $30 \%$ das idosas avaliadas apresentaram estalidos ${ }^{16}$.

$\mathrm{Na}$ avaliação mastigatória verificou-se que o padrão mastigatório predominante foi 0 bilateral simultâneo, seguido do unilateral direito e em seguida o padrão bilateral alternado com predomí- 
Tabela 9 - Distribuição de idosos de Instituição de longa permanência quanto à avaliação das funções estomatognáticas: mastigação

\begin{tabular}{lcccc}
\hline \multirow{2}{*}{ Avaliação das funções estomatognáticas } & \multicolumn{2}{c}{ Sim } & \multicolumn{2}{c}{ Não } \\
\cline { 2 - 5 } Mastigação & $\mathbf{N}$ & $\%$ & $\mathbf{N}$ & $\%$ \\
Bilateral alternada & 2 & 5,9 & 32 & 94,1 \\
Predomínio do lado direito & 4 & 11,8 & 30 & 88,2 \\
Predomínio do lado esquerdo & 1 & 2,9 & 33 & 97,1 \\
Bilateral simultânea & 9 & 26,5 & 25 & 73,5 \\
Simultânea com predomínio do lado direito & 5 & 14,7 & 29 & 85,3 \\
Bilateral simultânea com predomínio do lado esquerdo & 2 & 5,9 & 32 & 94,1 \\
Unilateral direita & 6 & 17,6 & 28 & 82,4 \\
Unilateral esquerda & 5 & 14,7 & 29 & 85,3 \\
Adaptada & 24 & 70,6 & 10 & 29,4 \\
Alterada & 1 & 2,9 & 33 & 97,1 \\
Lenta & 10 & 29,4 & 24 & 70,6 \\
Rápida & 10 & 29,4 & 24 & 70,6 \\
Ruidosa & 4 & 11,8 & 30 & 88,2 \\
Lábios fechados & 19 & 55,9 & 15 & 44,1 \\
Lábios abertos e/ou entreabertos & 10 & 29,4 & 24 & 70,6 \\
Interposição do lábio inferior & 4 & 11,8 & 30 & 88,2 \\
Participação exagerada da musculatura perioral & 28 & 82,4 & 6 & 17,6 \\
Movimentos rotatórios de mandíbula & 14 & 41,2 & 20 & 58,8 \\
Movimentos verticais de mandíbula & 30 & 58,8 & 14 & 41,2 \\
\hline
\end{tabular}

nio do lado direito. Dados que diferem dos achados em literatura, na qual se verificou que $50 \%(n=16)$ dos idosos avaliados apresentaram predominantemente um padrão unilateral, seguido do padrão bilateral alternado com $18,75 \%(n=6)$ e apenas 15,63 $(n=5)$ apresentaram o padrão bilateral simultâneo ${ }^{23}$. Em outro estudo realizado em 2005 com relação ao padrão mastigatório, foi visto que $36,8 \%(n=7)$ dos pesquisados apresentaram o padrão bilateral com predomínio de um dos lados; 31,6\% $(n=6)$ apresentaram padrão bilateral alternado; $21,1 \%(n=4)$ apresentaram o padrão unilateral e apenas $10,5 \%(n=2)$ realizaram o padrão bilateral simultâneo ${ }^{19}$.

Ainda na avaliação da mastigação foi percebido $70,6 \%(n=24)$ apresentaram uma mastigação adaptada, $11,8 \%(n=4)$, ruidosa, o que condiz com o que foi lido em literatura, quando é afirmado que a mastigação de indivíduos idosos também pode encontrar-se adaptada, ou seja, a mastigação desses voluntários se mostrou insuficiente, porém não se pode dizer que se trata de uma mastigação alterada, pois as mudanças apresentadas como: ruído; participação excessiva da musculatura perioral e movimentos verticais de mandíbula (padrão mastigatório bilateralmente simultâneo) são decorrentes do envelhecimento e da diminuição do tônus da musculatura envolvida, prejudicando assim a coordenação da lateralização do bolo alimentar e dos próprios movimentos mandibulares durante o ato mastigatório ${ }^{9,10}$. Também foi encontrado que $55,9 \%(n=19)$ mastigam com os lábios fechados, $82,4 \%$ ( $n=28$ ) com participação exagerada da musculatura perioral e $58,8 \%(n=30)$ com movimentos verticais da mandíbula, estando esse último inteiramente relacionado com o padrão mastigatório predominantemente encontrado neste estudo. Afirmase que a participação exagerada da musculatura perioral pode ser justificada pela tentativa do idoso perceber melhor o alimento dentro da boca e também por aumentar a pressão intra-oral preparando o bolo alimentar para ser deglutido ${ }^{27}$.

Em relação à deglutição observou-se que, no momento da avaliação assim como a mastigação, sofreu adaptações diante do envelhecimento de todas as estruturas envolvidas, principalmente no que diz respeito à participação exagerada da musculatura perioral. Na literatura foi encontrado que a contração exagerada da musculatura perioral durante a deglutição geralmente ocorre porque existe ou existiu a projeção anterior de língua. Sendo essa contração uma compensação para evitar que a língua se projete para fora. Esse mecanismo 
Tabela 10 - Distribuição de idosos de Instituição de longa permanência quanto à avaliação das funções estomatognáticas: deglutição e fonoarticulação

\begin{tabular}{lcccc}
\hline \multirow{2}{*}{ Avaliação das funções estomatognáticas } & \multicolumn{2}{c}{ Sim } & \multicolumn{2}{c}{ Não } \\
\cline { 2 - 5 } Deglutição & $\mathbf{N}$ & $\%$ & $\mathbf{N}$ & $\%$ \\
Normal & 1 & 2,9 & 33 & 97,1 \\
Alterada & 1 & 2,9 & 33 & 97,1 \\
Adaptada & 30 & 88,2 & 4 & 11,8 \\
Projeção anterior de língua & 13 & 38,2 & 21 & 61,8 \\
Projeção lateral de língua & 0 & - & 34 & 100 \\
Interposição do lábio inferior & 0 & - & 34 & 100 \\
Participação exagerada da musculatura perioral & 28 & 82,4 & 6 & 17,6 \\
Projeção de cabeça & 9 & 26,5 & 25 & 73,5 \\
Engasgo & 2 & 5,9 & 32 & 94,1 \\
Ruidosa & 16 & 47,1 & 18 & 52,9 \\
& & & & \\
Fonoarticulação & & & & \\
Lenta & 3 & 8,8 & 31 & 91,2 \\
Rápida & 10 & 29,4 & 24 & 70,6 \\
Escape de ar & 18 & 52,9 & 16 & 47,1 \\
Interposição de língua & 3 & 8,8 & 31 & 91,2 \\
Acúmulo de saliva nas comissuras labiais & 3 & 8,8 & 31 & 91,2 \\
Elevação de laringe normal & 10 & 29,4 & 24 & 70,6 \\
Elevação de laringe reduzida & 20 & 58,8 & 14 & 41,2 \\
Substituição & 20 & 58,8 & 14 & 41,2 \\
Omissão & 30 & 88,2 & 4 & 11,8 \\
Distorção & 19 & 55,9 & 15 & 44,1 \\
Imprecisão & 30 & 88,2 & 4 & 11,8 \\
\hline
\end{tabular}

pode ser encontrado também em pessoas que possuem o tônus diminuído, o que se torna comum ao idoso ${ }^{27}$.

Em estudo desenvolvido em 2004 foi observado um grande índice de idosas que apresentaram, durante a deglutição, compensação de anteriorização de cabeça em torno de $90 \%(n=9)^{16}$. Já na pesquisa atualmente desenvolvida, essa compensação foi observada em apenas $26,5 \%(n=9)$. No que diz respeito à deglutição ruidosa, afirma-se que esta ocorre por conta do excesso de força que o dorso da língua realiza. Geralmente, isso ocorre quando a ponta da língua se apresenta hipotensa e se posiciona em assoalho oral gerando uma dificuldade em realizar os movimentos ondulatórios necessários durante a deglutição ${ }^{24}$. Também se afirma que a presença do ruído durante a deglutição ocorre pela flacidez dos músculos da laringe e faringe ${ }^{28}$.

$\mathrm{Na}$ avaliação da fonoarticulação foi observado que $52,9 \%(n=18)$ apresentam escape de ar durante a fala, podendo esse dado ser justificado pela grande quantidade de idosos edêntulos pesquisados. Também foi visto que $58,8 \%(n=20)$ têm elevação de laringe reduzida, pois como é citado em literatura, com o avançar da idade as cartilagens laríngeas começam a se calcificar e perder elasticidade, assim como também ocorre a atrofia dos músculos da laringe ${ }^{28}$. Foi observado que $8,8 \%$ $(n=3)$ dos idosos apresentaram acúmulo de saliva durante a função de fonoarticulação, valor semeIhante ao encontrado em estudo desenvolvido em 2004, no qual se afirma que 4,6\% $(n=2)$ dos idosos apresentaram essa adaptação ${ }^{23}$. E por fim, foi possível observar que no momento da avaliação 58,8\% $(n=20)$ apresentaram substituição, 88,2\% $(n=30)$ apresentaram omissão, 55,9\% $(n=19)$ distorção e $88,2 \%(n=30)$ imprecisão, podendo esses números serem justificados por conta da falta de elementos dentários e pela própria diminuição do tônus dos órgãos fonoarticulatórios dificultando assim, a produção de determinados fonemas. 


\section{CONCLUSÃO}

A partir dos resultados obtidos nesta pesquisa, foi possível concluir que os idosos se adaptam durante o processo de mastigação a fim de manter sua dieta o mais próximo possível da realizada quando jovem. Assim como foi observado um padrão adaptado no processo mastigatório, constatou-se a presença de algumas compensações durante a deglutição permitindo uma alimentação segura. No entanto, torna-se interessante complementar a avaliação da deglutição com exames de videofluroscopia para maior fidedignidade dos achados. Também foi visto que as características fonoarticulatórias encontradas não comprometem a eficácia da comunicação dos idosos atualmente. Entretanto, é necessário que se tenha uma maior atenção a esse padrão de fala do idoso, pois essa função o mantém em contato com o mundo social, fator esse extremamente importante para uma boa qualidade de vida. Foi possível ainda observar que o atendimento ao idoso necessita ser realizado de forma interdisciplinar com o foco na atenção primária em uma perspectiva de integralidade das ações desenvolvidas. E que o fonoaudiólogo pode e deve fazer parte dessa equipe auxiliando na prevenção e/ou reabilitação de problemas relacionados às funções estomatognáticas.

\begin{abstract}
Purpose: to identify extant adaptations into stomatognathic functions of chew, deglutition and speech in aged people at a long permanence Institution. Methods: 34 aged people, of both genders, being 15 feminine and 19 masculine, with ages ranging from 65 to 88 years, took part in the study. Interviews were carried through containing questions for the aged people on general health and feeding and speech therapy evaluations were performed in order to characterize functions of chew, deglutition and speech. Results: as for chew, it was observed that the majority of aged people showed an adapted chew, with exaggerated participation of perioral muscles, heaves of jaw and predominant chew standard was bilateral simultaneous. As for deglutition, it was observed that this function as well as chew were an adapted form into the majority of volunteers majority, we also observed the occurrence of exaggerated participation of perioral muscles and noise during deglutition. In speech evaluation we noted that in more than $50 \%$ of the aged people, there was great air escape occurring during speaks, as well as a rise of reduced larynx and the presence of substitution, omission, distortion and imprecision. Conclusion: it was observed that aged people get adaptations during chew process in order to keep their possible diet next to when they were young. It was also noted that the studied aged people carry through some compensations during deglutition. The found speech characteristics do not limit severely aged people communication currently. It was still possible to observe that attendance to aged people needed to be carried through by an interdisciplinary team and that speech therapist can and must be part of this team assisting in preventing and/or rehabilitating issues related to stomatognathic functions.
\end{abstract}

KEYWORDS: Mastication; Deglutition; Speech; Aged

\section{REFERÊNCIAS}

1. Esteves B. O Brasil de cabelos brancos. Ciência Hoje. 1998; 23(137):18-21.

2. Lima-Costa MF, Veras R. Saúde pública e envelhecimento. Cad Saúde Pública. 2003; 19(3):700-1.

3. Camarano AA. Envelhecimento da população brasileira: uma contribuição demográfica. In: Freitas EV, PY L, Neri AL. Tratado de geriatria e gerontologia. Rio de Janeiro: Guanabara Koogan; 2002. p. 58-71.
4. Jacob Filho W. Envelhecimento e atendimento domiciliário. In: Duarte YA, Diogo MJD. Atendimento domiciliar: um enfoque gerontológico. São Paulo: Atheneu; 2000. p. 19-25.

5. Jordão Neto A. Aspectos psicossociais do envelhecimento. In: Russo IP. Intervenção fonoaudiológica na terceira idade. Rio de Janeiro: Revinter: 1999. p. 13-24.

6. Mitre El. Aspectos otorrinolaringológicos do idoso. In: Suzuki HS, organizador. Conhecimentos específicos para entender bem o paciente idoso. São José dos Campos: Pulso; 2003. p. 25-32. 
7. Ribeiro A. Aspectos biológicos do envelhecimento. In: Russo IP. Intervenção fonoaudiológica na terceira idade. Rio de Janeiro: Revinter; 1999. p. 1-11.

8. Bilton TL, Sustovich DR. Avaliação fonoaudiológica. In: Sustovich DR. Semiologia do idoso para o clínico. São Paulo: Sarvier; 1999. p. 81-4.

9. Couto EAB. Avaliação pelo fonoaudiólogo. In: Maciel A. organizador. Avaliação multidisciplinar do paciente geriátrico. Rio de Janeiro: Revinter; 2002. p. 157-77.

10. Perlman AL, Schulze-Derieus KS. Deglutition and its disorders: anatomy, physiology, clinical diagnosis, and management. San Diego: Singular Publishing Group; 1997.

11. Osterberg T, Tsuga K, Rothenberg E, Carlsson GE, Steen B. Masticatory ability in 80-year-old subjects and its relation to intake of energy, nutrients and food items. Gerodontol. 2002; 19(2):95-101.

12. Marshall TA, Warren JJ, Hand JS, Xie XJ, Stumbo PJ. Oral health, nutrient intake and dietary quality in the very old. J Am Dent Assoc. 2002; 133(10):1369-79.

13. Daniels SK, Corey DM, Hadskey LD, Legendre C, Priestly DM, Rosembek JC, et al. Mechanism of sequential swallowing during straw drinking in healthy young and older adults. J Speech Lang Hear Res. 2004; 47(1):33-45.

14. Duarte VG, Giacheti CM. Programa de atuação fonoaudiológica junto ao idoso institucionalizado. In: Lagrota MGM, César CPHAR. A fonoaudiologia nas instituições. São Paulo: Lovise; 1997. p. 17-27.

15. Jales MA, Cabral RR, Silva HJ, Cunha DA. Características do sistema estomatognático em idosos: diferenças entre instituição pública e privada. Rev CEFAC. 2005; 7(2):178-87.

16. Amaral AKFJ. Presbifagia: o envelhecimento da deglutição de idosas do conviver geriátrico [monografia]. João Pessoa (PB): Universidade Federal da Paraíba; 2004.

17. Massey BT, Shaker R. Introduction to the field of deglutition and deglutition disorders. In: Perlman AL, Schulze-Delrieu KS. Deglutition and its disorders: anatomy, physiology, clinical diagnosis and management. San Diego: Singular Publishing Group; 1997.p.1-14.

18. Duthie EH, Katz PR. Geriatria prática. Rio de Janeiro: Revinter; 2002.

19. Wendy A. In: Edmund H, Duthie Jr. MD, Katz PR. Geriatria prática. Rio de Janeiro: Revinter; 2002. p.380.

20. Rademaker AW, Pauloski BR, Colangelo LA, Logemann JA. Age and volume effects on liquid swallowing function in normal women. J Speech Lang Hear Res. 1998; 41(2):275-84.

21. Suzuki HS. O entardecer da deglutição: um estudo sobre modificações nos hábitos da rotina alimentar $x$ fisiologia da deglutição do idoso normal. [monografia] São Paulo (SP): CEFAC - PósGraduação em Saúde e Educação; 1997.

22. Marchesan IQ. Distúrbios da motricidade oral. In: Russo IPC. Intervenção fonoaudiológica na terceira idade. Rio de Janeiro: Revinter; 1999. p. 83-100.

23. Castro SAFN, Santos AC, Gonçalves LHT. A fala dos idosos: modificações associadas ao envelhecimento do sistema estomatognático. Rev Bras Ciênc Envelhec Hum. 2004; 1(2): 41-51.

24. Palmer JB, DuChane AS. Rehabilitation of swallowing disorders in the elderly. In: Felsenthal G, Garrison SJ, Steinberg FJ. Rehabilitation of the aging and older patient. Baltimore: Willians and Wilkins; 1994. p. 275-87.

25. Marcenes W, Steele JG, Sheiham A, Walls AWG. The relationship between dental status, food selection, nutrient intake, nutritional status, and body mass index in older people. Cad Saúde Pública. 2003; 19(3):809-16.

26. Shanley C, O'Loughlin G. Dysphagia among nursing home residents: an assessment and management protocol. J Gerontol Nurs. 2000; 26(8):35-48.

27. Marchesan IQ. Fundamentos em fonoaudiologia: aspectos clínicos da motricidade Oral. Rio de Janeiro: Lovise; 1998.

28. Alencar SC, Pereira SMS, Cysne C. O processo de envelhecimento e as características da função mastigatória. Rev CEFAC. 2003; 5(3):247-50.

RECEBIDO EM: 08/06/2007

ACEITO EM: 15/06/2008

Endereço para correspondência:

Renata Milena Lima

Rua Barão de Souza Leão, 514 BI-B ap. 604

Recife - PE

CEP: $51030-300$

E-mail: re_fono@ @otmail.com 